\title{
MICROCONTROLLER METERING CONVERTER OF CAPACITANCE WITH VOLTAGE-CONTROLLED RC-GENERATOR
}

\author{
Aleksandr Vostrukhin ${ }^{1}$, Elena Vakhtina ${ }^{2}$, Sergey Bondar ${ }^{2}$, Lukash Tomashik ${ }^{3}$ \\ ${ }^{1}$ Stavropol Technological Institute of Service, Russia; ${ }^{2}$ Stavropol State Agrarian University, Russia; \\ ${ }^{3}$ NUPSESO, a.s., Slovakia \\ avostrukhin@yandex.ru, eavakhtina@yandex.ru, bond_sn@mail.ru, tomasik.lukas@gmail.com
}

\begin{abstract}
In agriculture, the moisture content of seeds, air and other materials is measured using capacitive sensors. The voltage (metering voltage, MV) that varies with a certain frequency is supplied to a capacitive sensor.The MV frequency can be either fixed or variable, depending on the measured capacitance changing. In the last case, additional errors occur during the measurement.For example, for Honeywell's HCH-1000 air humidity sensors, changing the MV frequency from 50 to $100 \mathrm{kHz}$ leads to a humidity measurement error within $10 \%$. The main parameters of these sensors are given for the frequency of $20 \mathrm{kHz}$ in the datasheet. It is known that the seed permittivity depends on the MV frequency of the capacitive sensor, in the cavity of which these seeds are located. Therefore, the task is to control the MV frequency.Microcontroller-controlled process of capacitance-to-binary code conversion is considered in the paper. Capacitance-to-frequency conversion is carried out using the NE555 chip operating in the auto-generator mode. Arduino's microcontroller measures the frequency at NE555 output, then its deviation from the value set is determined and an impact in the form of voltage is formed in order to eliminate this deviation. The result of the conversion is a binary code equivalent to the effect generated to eliminate the deviation. Thus, the MV frequency is maintained at a predetermined level.Experimental studies were carried out in a capacitancechange range from 200 to $400 \mathrm{pF}$. This range was selected because, when the air humidity varies from 0 to $100 \%$, the range of the HCH-1000 sensors capacitance changes from 280 to $380 \mathrm{pF}$. Microcontrollers allow us to solve the tasks of converting physical quantities into binary code quite simply and effectively. Binary code allows performing any arithmetic operations, which in turn increases the measurements accuracy. It does not require bulky hardware. All devices necessary for organizing the conversion process can be placed on a single semiconductor crystal in the future.
\end{abstract}

Keywords: binary code, duty ratio, frequency, NE555, pulse-width modulator.

\section{Introduction}

Microcontroller metering converter of capacitance (MMCC) was considered in [1]. The MMCCbased USB-moisture meter for seeds of agricultural crops was developed.The MMCC output signal is the frequency that depends on the capacity of the sensor in the cavity of which seeds are located. The sensor capacity depends mainly on the seed moisture content. The MMCC output frequency is equal to the frequency of the electric field (EF) difference between the sensor's plates.It is known that the dielectric permittivity of seeds depends on the frequency of EF change.To increase the accuracy of measurements, it is necessary to stabilize this frequency or take its change into account.MMCC, which allows by program automatically control the EF frequency of the capacitive sensor that is discussed in the article.This solution allows pieces of metering at several frequencies, which can improve the accuracy of measurements.Studies of moisture measurement methods for bulk materials at different frequencies of EP have been considered in reference [2].

It should be noted that the capacitive air humidity sensors must operate at a certain frequency.For example, in the datasheet of Honeywell Corporation, the characteristics of capacitive air humidity sensors are considered for only $20 \mathrm{kHz}$ frequency [3].

\section{Materials and methods}

The NE555 chip appearance on the market makes it possible to realize the capacitance-tofrequency metering convertor in a sufficiently simple way, it promoted the development of digital capacitance measuring devices. However, known devices use frequency dependence on the measured capacitance, which in some cases leads to a decrease in their accuracy.Let us consider the implementation of the automatic stabilization method of the MMCC frequency using the NE555 chip.

Figure 1 shows the MMCC circuit diagram, which consists of two measuring subsystems: the NE555-based capacitance-to-frequency converter (CFC) and the Arduino-based frequency-to-code (digit) converter (FDC). MMCC output information signal is transmitted via USB interface to the PC. 
The capacitive sensor $C x$ is included (Figure 1) as a time-setting element of the RC generator built on the NE555 chip [4]. The NE555 output signal (pin OUT) goes to the Arduino Uno microcontroller system (pin D5), where it is processed, and enters the PC in the final result form. The Arduino microcontroller platform is widely known in the world, continuously improved (https://www.arduino.cc/) and is used in engineering education [5], scientific researches [6], and also in applied developments [7].The MMCC studies were carried out using a capacitor of variable capacitance as the $C x$, which appearance is shown in Figure 2. The resolution of this capacitor is $0.2 \mathrm{pF}$, the capacitance range is from $50 \mathrm{pF}$ to $1150 \mathrm{pF}$.

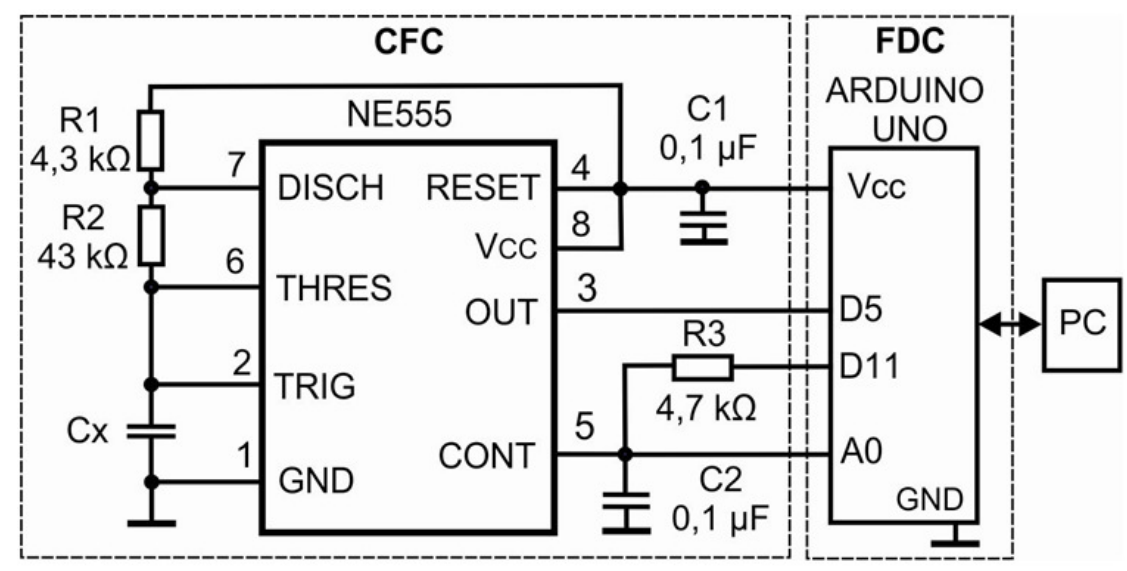

Fig. 1. MMCCCircuit Diagram

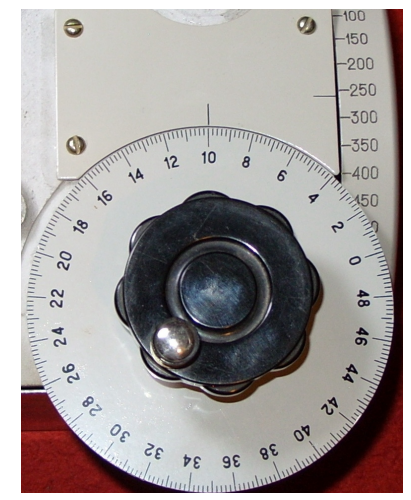

Fig. 2. Capacitor of variable capacitance

The using of NE555 for CFC building is convenient in that the capacitance $\mathrm{Cx}$ is converted to the frequency of rectangular pulses. All microcontrollers contain, as a rule, at least two hardware binary counters of rectangular pulses. This allows organizing a fixed time interval (a gate time) on one counter, and on the other - to count the pulses received at its input during this time interval.The NE555 mathematical model is included in the popular at engineering faculties Multisim http://www.ni.com/multisim simulation environment for designing of electronic devices [1].

The main characteristic of the metering converter is a conversion characteristic, which determines the dependence of the output signal on the input signal. We are interested in the dependence of the binary code $N$ on the capacity $C x$. It is desirable that the conversion characteristic be linear. The CFC subsystem (Figure 1) is a typical RC-generator circuit operating in the "Astable Operation" mode [4]. If a variable voltage is applied to the CONT pin of the NE555 chip, then the frequency of the EFof the capacitive sensor $C x$ can be controlled. The frequency of the EF is equal to the frequency of the $F_{C F C}$ at the NE555 output.

It is known that the pulse-width modulation (PWM) signal can be converted into a DC voltage using an RC filter. Changing the duty ratio $(G)$ of the PWM signal results in a proportional change in voltage across the $\mathrm{RC}$ filter output.Thus, it is possible to obtain voltage for the $F_{C F C}$ control. We agree to call this voltage - the controlling $(V c)$. Resistor $R_{3}$ and capacitor $C_{2}$ (Figure 1) form the RCfilter.

$F_{C F C}$, in general, is defined according to equation (1):

$$
F_{C F C}=\frac{k}{\left(R_{1}+2 \cdot R_{2}\right) \cdot C_{x}},
$$

where $k$-coefficient that depends on the specific chip, for example, for a Philips chip $k=1.49$ [4], and Texas Instruments $k=1.44$ [8].

The MMCC algorithm that implements the controlled conversion process is presented below. A "given frequency" $\left(F_{0}\right)$ concept is introduced into the algorithm - it is the value of the frequency that the MMCC should support, when $C x$ is changing. When analyzing this algorithm (step 3), it should be taken into account that as $G$ increases, the $F_{C F C}$ frequency decreases, as $G$ decreases, the $F_{C F C}$ frequency increases. 


\section{The MMCC algorithm}

The algorithm consists of the following steps:

1. generate a PWM signal for $G=0.5$;

2. measure the $F_{C F C}$;

3. if $F_{C F C}<F_{0}$, then go to block 4 , if $F_{C F C}>F_{0}$, then go to step 6;

4. decrement $G$;

5. display $G$ on PC and go to step 2;

6. increment $G$;

7. display $G$ on PC and go to step 2 .

The authors used a functionally-oriented method for software development - the decomposition of the problem solution into separate modules that perform certain functions.

Arduino Uno is a microcontroller board based on the ATmega328P 8-bit microcontroller of Atmel Corporation. This microcontroller contains three timers/counters: one TC1 -the 16-bit and two TC0, TC2 - the8-bit [9].

The TC1 timer/counter is used to implement the frequency meter module. NE555 pin OUT is connected to the counting input TC1 - pin D5 (Figure 1). Resistor $R_{3}$ is switched out from Arduino Uno in this case by means of switching the D11 digital pin to a high-resistance state. The voltage at the pin CONT is $2 / 3 V c c$, where $V c c=5 \mathrm{~V}$, is the supply voltage of the microcontroller. A program to implement the function of a frequency meter has been developed.The non-linear dependence of $F_{C F C}$ (pin OUT) on $C x$, if there is no control, is shown in Figure 3.The capacitance $C x$ was obtained by means of a calibrated capacitor of variable capacitance shown in Figure 2.

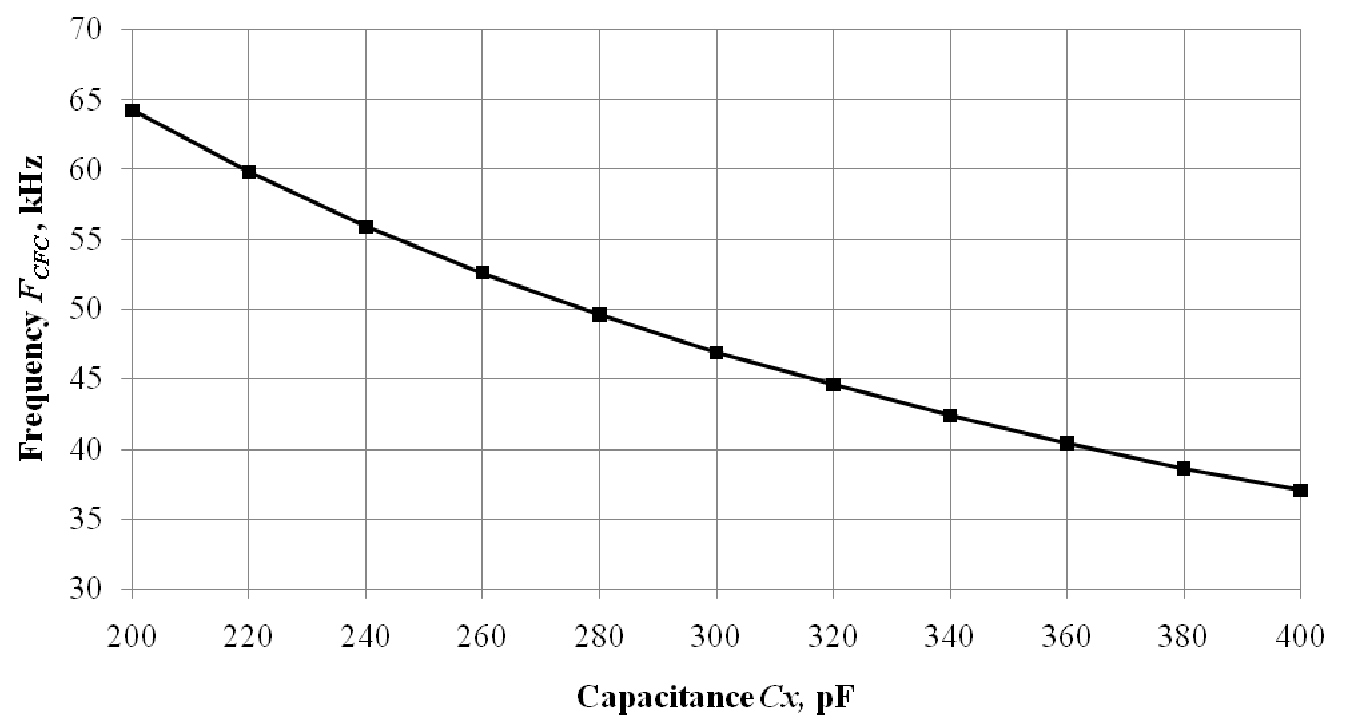

Fig. 3. Dependence of $\boldsymbol{F}_{C F C}$ on $\boldsymbol{C x}$, if there is no control

Together with the RC filter, the PWM module implements the function of a digital-to-analog converter (DAC) to form a control voltage $V c$. The PWM signal from the PWM output (pin D11) is provided to the RC filter input (Figure 1). The smoothed DC voltage $V c$ of the RC filter output is applied to the CONT input of the NE555 chip.

PWM is implemented based on the TC2 timer/counter. The TC0 timer/counter is used by the IDE Arduino library to realize time delays and the frequency meter program. We select the "Fast PWM Mode" that gives the maximum frequency of the PWM signal at the RC filter input and, consequently, $V c$ with smaller pulsations. The PWM frequency for the output can be calculated by equation (2):

$$
f_{P W M}=\frac{f_{c l k}}{n \cdot 256},
$$

where $\quad n$-variable that represents the pre-scale factor $(1,8,32,64,128,256$, or 1024) [9]. 
The Arduino UNO microcontroller operates at a clock frequency of $16 \mathrm{MHz}$ according to equation (2), the PWM signal frequency will be $63 \mathrm{kHz}$.

\section{Results and discussion}

To research in the MMCC, it is necessary to know the dependence of $F_{C F C}$ on $V c$.Therefore, an experiment was conducted, in which a 10-bit analog-to-digital converter (ADC) of the ATmega328P microcontroller was used to measure $V c$. Figure 1 shows that the CONT input is connected to the ADC input - this is pin A0. A program has been developed that makes it possible to obtain the dependence of $F_{C F C}$ on $V c$. The results of this program are given in Table 1. After each $N$ incrementing, four discretely changing values: $N, G, V c$ and $F_{C F C}$ are output to the PC monitor. Dependence $V c$ from $N$ is linear. Theduty ratio is defined byequation (3):

$$
G=N / 255 \text {, }
$$

where 255 - decimal equivalent of the maximum 8-bit binary code.

The $F_{C F C}$ data are given for three fixed values of the $C x$ capacitance.

$G, V c$ and $F_{C F C}$ dependencies on $N$

Table 1

\begin{tabular}{|c|c|c|c|c|c|c|c|c|c|c|c|c|}
\hline $\boldsymbol{N}$ & $\mathbf{0}$ & $\mathbf{2 5}$ & $\mathbf{5 0}$ & $\mathbf{7 5}$ & $\mathbf{1 0 0}$ & $\mathbf{1 2 5}$ & $\mathbf{1 5 0}$ & $\mathbf{1 7 5}$ & $\mathbf{2 0 0}$ & $\mathbf{2 2 5}$ & $\mathbf{2 5 0}$ & $\mathbf{2 5 5}$ \\
\hline $\boldsymbol{G}$ & 0.0 & 0.098 & 0.196 & 0.294 & 0.392 & 0.490 & 0.588 & 0.686 & 0.784 & 0.882 & 0.980 & 1.0 \\
\hline $\boldsymbol{V} \boldsymbol{c}, \mathbf{V}$ & 1.98 & 2.18 & 2.37 & 2.57 & 2.77 & 2.98 & 3.18 & 3.37 & 3.57 & 3.77 & 3.97 & 4.00 \\
\hline $\begin{array}{c}\boldsymbol{F}_{\boldsymbol{C F C}}, \mathbf{k H z} \\
(\boldsymbol{C} \boldsymbol{x}=\mathbf{2 0 0 p F})\end{array}$ & 90.8 & 87.2 & 83.4 & 79.5 & 75.5 & 71.5 & 67.4 & 62.5 & 59.2 & 54.9 & 50.6 & 49.9 \\
\hline $\begin{array}{c}\boldsymbol{F}_{\boldsymbol{C F C} \boldsymbol{~}}, \mathbf{k H z} \\
(\boldsymbol{C} \boldsymbol{x = 3 0 0} \mathbf{~ p F})\end{array}$ & 67.3 & 64.4 & 61.8 & 58.6 & 55.6 & 52.5 & 49.4 & 46.3 & 43.2 & 40.0 & 36.8 & 36.3 \\
\hline $\begin{array}{c}\boldsymbol{F}_{\boldsymbol{C F C}}, \mathbf{k H z} \\
(\boldsymbol{C} \boldsymbol{x = 4 0 0} \mathbf{~ p F})\end{array}$ & 53.5 & 51.2 & 48.8 & 46.4 & 43.9 & 41.5 & 39.0 & 36.5 & 34.0 & 31.4 & 28.9 & 28.5 \\
\hline
\end{tabular}

A program that implements the MMCC algorithm described above has been developed. The results of this program for two frequencies $F_{C F C}=50 \mathrm{kHz}$ and $F_{C F C}=53 \mathrm{kHz}$, presented in Figure 4, show that the binary code $\mathrm{N}$ dependence on $\mathrm{Cx}$ over the entire range of $\mathrm{Cx}$ variation is linear.

It was experimentally established that the coefficient $k$ depends on $F_{C F C}$. Therefore, it is necessary to determine $k$ for the frequency range of 10 to $100 \mathrm{kHz}$. For these frequencies, Haneyuwell Corporation provides the main characteristics of the HCH-1000 series capacitive sensors [3].From equation (1) we find $k$ by formula (4):

$$
k=F_{C F C} \cdot\left(R_{1}+2 \cdot R_{2}\right) \cdot C x .
$$

The $F_{C F C}$ frequency is changed by changing $C x$ and measured using an oscilloscope. The results of $F_{C F C}$ meterings and calculations of $k$ are shown in Table 2.

Table 2

Dependence of $\boldsymbol{k}$ coefficient on $\boldsymbol{F}_{C F C}$ frequency

\begin{tabular}{|c|c|c|c|c|c|c|c|c|c|c|}
\hline $\boldsymbol{F}_{\boldsymbol{C F C}}, \mathbf{k H z}$ & 10 & 20 & 30 & 40 & 50 & 60 & 70 & 80 & 90 & 100 \\
\hline $\boldsymbol{k}$ & 1.54 & 1.44 & 1.35 & 1.28 & 1.2 & 1.14 & 1.05 & 0.96 & 0.91 & 0.90 \\
\hline
\end{tabular}

It is established that control is performed only in the frequency range of 50 to $53 \mathrm{kHz}$. At frequencies below 50 and above $53 \mathrm{kHz}$, the binary code goes beyond 8-bit. To go to other frequencies, the change of $R_{1}$ and $R_{2}$ values is needed (Figure 1). We can recommend the following procedure for calculating $R_{1}$ and $R_{2}$ depending on the given $F_{C F C}$ frequency.

For example, $F_{C F C}=20 \mathrm{kHz}$ is needed. We determine the average value of $C x$ for a given frequency: $C=\left(C_{\min }+C_{\max }\right) / 2$, where $C_{\min }$ and $C_{\max }$ are the minimum and maximum values of $C x$, which are given for $\mathrm{HCH}-1000$ Series Capacitive Sensors in [3]. Then from equation (1) we obtain (5): 


$$
R_{1}+2 \cdot R_{2}=\frac{k}{F_{C F C} \cdot C} .
$$

We find: $R_{1}+2 \cdot R_{2}=1.44 / 20 \cdot 300=240 \mathrm{k} \Omega$. If the resistance is in $\mathrm{k} \Omega$, and the capacitance in $\mathrm{pF}$, then the time constant will be in ns.

It is known that $R_{2}=10 \cdot R_{1}$, then, $R_{1}+2 \cdot 10 \cdot R_{1}=21 \cdot R_{1}=240 \mathrm{k} \Omega$, hence $R_{1}=240 / 21 \approx 11,4 \mathrm{k} \Omega$, and $R_{2}=10 \cdot 11.4 \approx 114 \mathrm{k} \Omega$. We find the nearest resistance values from the standard series: $R_{1}=11 \mathrm{k} \Omega$ and $R_{2}=110 \mathrm{k} \Omega$. We get $R_{1}+2 \cdot R_{2}=11+2 \cdot 110=231 \mathrm{k} \Omega$. We check the calculation by equation (1): $F_{C F C}=1.44 / 231 \cdot 300=20779 \mathrm{~Hz} \approx 21 \mathrm{kHz}$. We accept $R_{1}=11 \mathrm{k} \Omega, R_{2}=110 \mathrm{k} \Omega$ and embed these resistors in the circuit shown in Figure 1.

Dependences of $N$ on $C x$ for three frequencies of 20,21 and $22 \mathrm{kHz}$ were experimentally obtained. In the case of $F_{C F C}=20 \mathrm{kHz}$ and $C x=200 \mathrm{pF}$, the binary code went beyond the 8-bit code. Dependences of $N$ on $C x$ for two frequencies $F_{C F C}=21 \mathrm{kHz}$ and $F_{C F C}=21 \mathrm{kHz}$ are shown in Figure 4. Thus, the $R_{1}$ and $R_{2}$ calculationis correct.

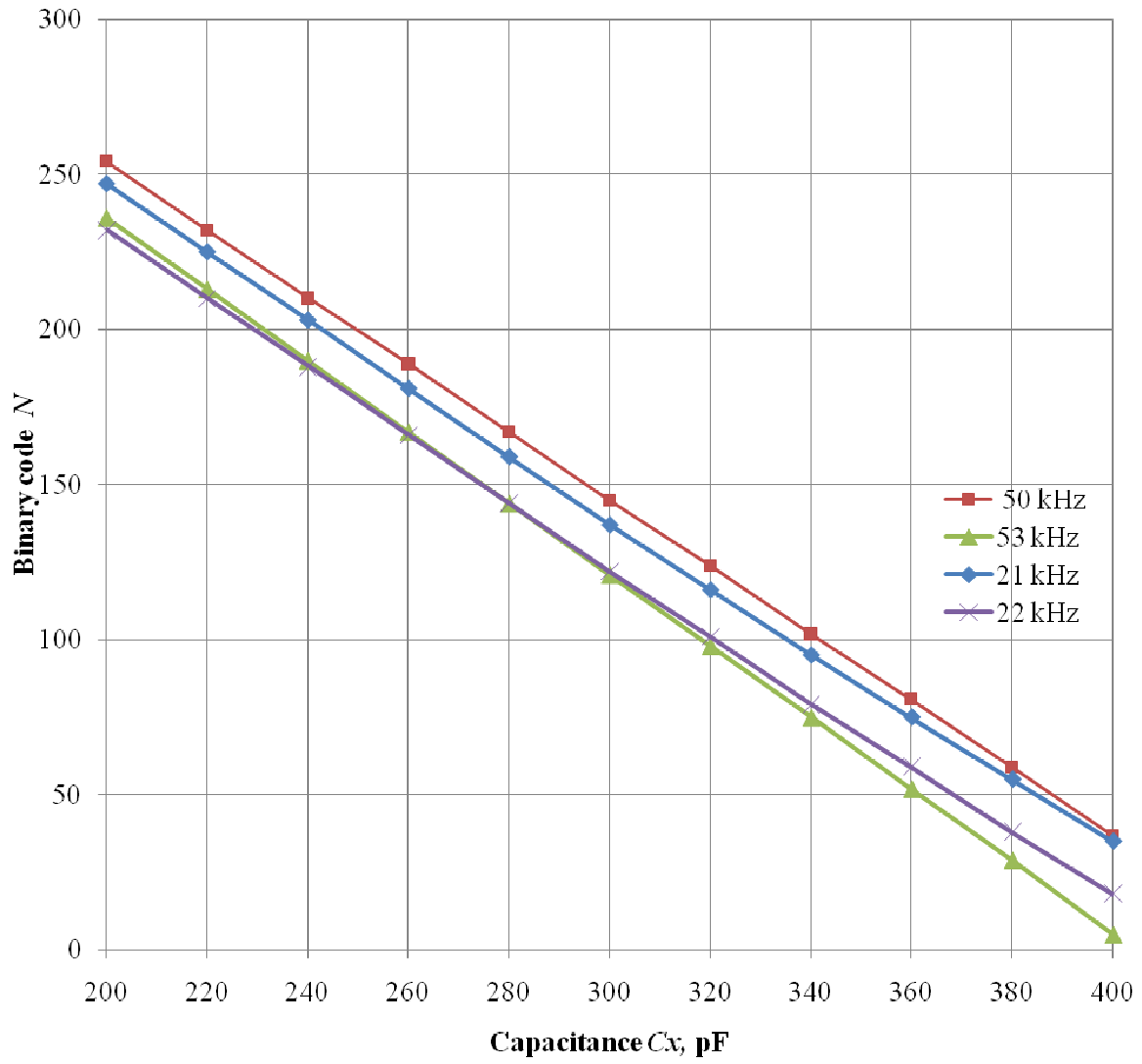

Fig. 4. Dependences of binary code $N$ on $\mathrm{Cx}$ for four fixed frequencies

\section{Conclusions}

1. An algorithm that implements a program-controlled method of the capacitance conversion into a binary code is proposed and investigated.

2. It has been experimentally established that for a fixed frequency of a voltage-controlled RC generator, the dependence of the MMCC output signal on the converted capacitance is linear.

3. An example of determining the coefficient $k$ for Philips NE555 chip, which can be used to find $k$ for NE555chips from other manufacturers, is given.

4. A technique for calculating time-setting resistances for the most common range of HCH-1000 Series Capacitive Sensors is proposed and tested.

5. The MMCC hardware implementation MMCC has been developed.

6. The developed modules for the MMCC program, implementing separate conversion processes, enable the most complete analysis and synthesis of MMCC in solving engineering and scientific 
problems requiring organization of measurement and control systems for physical quantities controlled by capacitive sensors.

\section{References}

[1] Vostrukhin A., Vakhtina E., MastepanenkoM., Gabrielyan Sh. Dielectric USB-Moisture Meter for seeds of agricultural crops. Proceedings of 8th International Scientific Conference "Rural Development 2017", November 23-24, 2017 Aleksandras Stulginskis University, Lithuania, [online] [31.03.2011]. Available at: http://conf.rd.asu.lt/index.php/rd/article/view/407

[2] Дубров Н.С., Кричевский Е.С., Невзлин Б.И.. Многопараметрические влагомеры для сыпучих материалов (Multiparameter moisture meters for bulk materials). Moscow: Mechanical Engineering, 1980. 144 p. (In Russian).

[3] HCH-1000 Series Capacitive Humidity Sensors Datasheet. [online] [24.02.2018]. Available at http://html.alldatasheet.com/html-pdf/230829/HONEYWELL/HCH-1000/487/1/HCH-1000.html.

[4] NE555 Datasheet. [online] [25.02.2018]. Available at: http://www.datasheetspdf.com/pdf/454331/Philipss/NE555/1.

[5] Vostrukhin A., Vakhtina E. Studying Digital Signal Processing on Arduino Based platform. Proceedings of International scientific conference "Engineering for Rural Development", Volume 15, May 25-27, 2016, Jelgava, Latvia. [online] [30.05.2016]. Available at: http://www.tf.llu.lv/conference/proceedings2016/Papers /N043.pdf

[6] Akhmarch A.R., Lazarescu M.T., Tarig O.B., Lavagno L. A Tagless Indoor Localization System Based on Capacitive Sensing Technology. Sensors, vol. 16, 1448; doi: 10.3390/s16091448, 2016.

[7] Blum J. Exploring Arduino: Tools and Techniques for Engineering Wizardry. Wiley, 2013. 384 p.

[8] NE555 Datasheet. [online] [25.02.2018]. Available at https://www.ti.com/lit/ds/symlink/se555.pdf

[9] ATmega328/P. Datasheet complete. [online] [04.03.2018]. Available at http://mkprog.ru/wpcontent/uploads/2017/09/ATmega328-328P_Datasheet.pdf. 